\title{
A COMPLETELY INTEGRABLE HAMILTONIAN SYSTEM ASSOCIATED WITH LINE FITTING IN COMPLEX VECTOR SPACES
}

\author{
BY ANTHONY M. BLOCH
}

Introduction. Over the past decade there has been a great deal of work on the explicit integration of completely integrable Hamiltonian systems. (See Adler and Van Moerbeke [1], McKean [9], Moser [11], Mumford [13].) Among the systems that have been studied are the free $n$-dimensional rigid body, the Euler-Poisson equations, geodesic flow on an ellipsoid, Neumann's equations, the Toda lattice, and Nahm's equations. The flows of these systems can be shown to linearize on the real part of the Jacobi variety of an algebraic curve associated with the system (see Adler and Van Moerbeke [1], Griffiths [7]).

Now, all of the above systems, except Nahm's equations, which arise in the theory of monopoles, come from problems in classical mechanics.

Here we present and explicitly integrate a completely interable Hamiltonian system that arises in a statistical problem - the fitting of lines to a data set in a complex vector space.

Remarkably, this system fits into the general scheme for integrating systems of "spinning top and ellipsoid type" developed by Moser [11] and Adler and Van Moerbeke [1]. Further, both the considered integrals and the flow have an interesting statistical meaning.

1. Let $\mathbb{C}^{n}$ denote complex $n$-dimensional euclidean space with orthonormal basis $e_{i}, i=1, \ldots, n$. Let $x_{i}=\sum_{j=1}^{n} \lambda_{i j} e_{j}, i=1, \ldots, p$ be $p$ data points. Then the total least squares estimate (see Golub and Van Loan [6]) of a $d$-plane fitted to the data set is given by minimizing the total perpendicular distance of the points from the plane. (In the case of lines this corresponds to the first principal component of the data (see Kendall [8]).)

The distance function is given by

$$
H(Q)=\operatorname{Tr} C(I-Q)=\operatorname{Tr} C P,
$$

where $P=I-Q, Q=$ orthogonal projection matrix of $\mathbb{C}^{n}$ onto the $d$-plane, and $C$ is a matrix with entries $c_{k j}=\sum_{i} \lambda_{\overline{i j}} \lambda_{i k}$.

Now let $G_{C}(d, n)=$ the complex Grassmanian of $d$-planes in $n$-space, $u(n)=$ the Lie algebra of the unitary group $U(n), h(n)=n \times n$ Hermitian matrices. Note that $P, C \in h(n), P$ having rank $n-d$. Remarkably then, $H$ is the restriction of a linear functional to $C_{\mathbb{C}}(d, n)$, viewed as an adjoint orbit of $u(n)$ (with rank $n-d$ matrices). Further, since $U(n)$ is compact, adjoit and coadjoint orbits may be identified via the Killing form. Then $H$ may be regarded as Hamiltonian on an adjoint orbit of $u(n)$ with the inherited Kostant-Kirilov symplectic structure.

Received by the editors November 13, 1984.

1980 Mathematics Subject Classification. Primary.

(C) 1985 American Mathematical Society $0273-0979 / 85 \$ 1.00+\$ .25$ per page 
Now $H$ lies in a maximal toral subalgebra of $u(n)$. Hence, we have a torus action on $G(d, n)$, and the Hamiltonian $H$ has an almost periodic vector field. The dimension of the image of the moment map (see Atiyah [2], for example) arising from this action is $n-1$ (since there is a trace condition on $Q$ ). Thus in the case $d=1$, where the adjoint orbit is $C P^{n-1}$, the system is completely integrable.

We now proceed to analyze this case in detail, carrying out the explicit integration. We remark that proof of integrability via the moment map is far from providing an explicit solution of the Hamiltonian flow.

2. Integration is carried out by describing the motion of a Lax pair with parameter and deriving action angle coordinates via the associated algebraic curve.

Now consider the Hamiltonian $H(Q)=\operatorname{Tr} C Q$. We have

THEOREM 1. For $H(Q)=\operatorname{Tr} C Q$, the Hamiltonian equations on an adjoint orbit of $u(n)$ are given by the Lax equation

$$
\dot{Q}=[Q, C] \text {. }
$$

To (1) we may associate the Lax equation with parameter

$$
(Q \dot{+} \xi C)=[Q+\xi C, C] \text {. }
$$

ProOF. $H$ is a left invariant function on the tangent bundle $T U(n)$ of $U(n)$. (1) then follows from a theorem of Raitu [14]. We see that (2) reduces to $(1)$.

With our system we then associate the algebraic (spectral) curve

$$
\operatorname{det}(Q+\xi C-\eta I)=0 \text {. }
$$

An alternative and instructive viewpoint is to write $H(Q)=\operatorname{Tr} C Q^{2}$ and regard our problem as a limiting (highly degenerate case) of the generalized rigid body on $U(n)$. The Lax equations then take the form

$$
Q=[Q, M], \quad M=C Q+Q M,
$$

and

$$
(Q \dot{+} \xi C)=\left[Q+\xi C, M+\xi C^{2}\right]
$$

(see $[\mathbf{4}]$ and $[\mathbf{1 5}]$ ).

3. One method for determining a basis for the integrals is to consider the coefficients of $\xi$ in the functions $\operatorname{Tr}(Q+\xi C)^{k}, k=2, \ldots, n$. For $H(Q)=$ $\operatorname{Tr} C Q^{2}$ defined on a generic adjoint orbit, this gives $\sum_{i=2}^{n} i=n(n-1) / 2$ nontrivial integrals, precisely half the dimension of a generic adjoint orbit in $u(n)$. These can be shown to be independent via a counting argument viewing $u(n)$ as the normal subalgebra of $\operatorname{sp}(n, \mathbb{C})$ (see $[10])$. 
In our limiting case $Q^{2}=Q$, the number of integrals is reduced but gives us

THEOREM 2. $A$ basis for the integrals of $H=\operatorname{Tr} C Q$ is given by $\operatorname{Tr} C Q$, $\operatorname{Tr} C^{2} Q, \ldots, \operatorname{Tr} C^{n-1} Q$ (for generic $C$ ).

An interesting statistical interpretation of the integrals is to view $Q$ as a density operator on $n$-dimensional Hilbert space. Then the integrals are the expected values of $C^{k}$ (in the quantum mechanical sense) or the higherdimensional moments of $C . \operatorname{Tr}(C Q)^{k}$ also occur as integrals. These too have an interesting statistical meaning (see $[\mathbf{4}]$ ).

4. Our main theorem is

THEOREM 3. Let $Q=\left(q_{\imath \jmath}\right)$ be written $Q=z \otimes \bar{z}, z=\left(z, \ldots, z_{n}\right) \in \mathbb{C}^{n}$, $\sum_{i=1}^{n}\left|z_{i}\right|^{2}=1, z_{1}=x_{i}+y_{i}$, and diagonalize $C, C=\operatorname{diag}\left(c_{1} \cdots c_{n}\right)$. Let $H(Q)=\operatorname{Tr} C Q$. Then the algebraic curve associated with $H$ is given by

$$
\xi=\sum_{i} \frac{x_{i}^{2}+y_{i}^{2}}{\eta-c_{\imath}},
$$

and the Hamiltonian flow on an adjoint orbit of $u(n)$, due to $H$, is given by

$$
q_{\imath j}(t)=\alpha_{i j} \exp \left(i\left(c_{j}-c_{i}\right) t+\beta_{\imath \jmath}\right),
$$

where $\alpha_{i j}, \beta_{i j}$ are constants.

ProOF. We use the arguments of Moser on rank 2 perturbations $[\mathbf{1 1}, \mathbf{1}]$. Let $R_{x x}=x \otimes x, \Gamma_{y y}=y \otimes y, \Gamma_{x y}=x \otimes y-y \otimes x$, and $\Gamma_{z \bar{z}}=z \otimes \bar{z}=Q$. Then consider the complex rank 1 ("real rank 2") perturbation of $C$ given by

$$
L=L(x, y)=C \xi+\Gamma z \bar{z}=C \xi+\Gamma_{x x}+\Gamma_{y y}+i \Gamma_{x y} .
$$

The eigenvalues of $L$ are in involution with respect to the symplectic structure $\sum_{j}^{n} d y_{\jmath} \wedge d x_{j}$. Now from the Weinstein-Aronsjan formula,

$$
\frac{\operatorname{det}(\eta-L)}{\operatorname{det}(\eta-C)}=1-\operatorname{Tr} W_{n}=1-\phi_{n}
$$

where

$$
\begin{gathered}
W_{\eta}=\left(\begin{array}{cc}
Q_{\eta}(x) & Q_{\eta}(x, y) \\
Q_{\eta}(x, y) & Q_{\eta}(y)
\end{array}\right)\left(\begin{array}{cc}
1 & i \\
-i & 1
\end{array}\right), \\
Q_{\eta}(x, y)=\left\langle R_{\eta} x, y\right\rangle, \quad Q_{\eta}(x)=Q_{\eta}(x, x), \\
R_{\eta}=(\eta I-C)^{-1}, \quad \phi_{n}=\operatorname{Tr} W_{\eta} .
\end{gathered}
$$

Note that, in contrast to Moser, $\operatorname{det} W_{\eta}=0$ here. For $\eta, \eta^{\prime}$ in the resolvent set of $C,\left\{\phi_{n}, \phi_{n}^{\prime}\right\}=0$ (see $[\mathbf{1 1}]$ ).

Thus the spectral curve is given by

$$
\operatorname{det}\left(\eta-C+\xi^{-1} \Gamma_{z \bar{z}}\right)=\operatorname{det}(\eta-C)\left(1-\phi_{\eta}\right)=0,
$$

where $\phi_{\eta}=\xi^{-1} Q_{\eta}(x)+\xi^{-1} Q_{\eta}(y)$. Since $C$ is diagonal, we find

$$
\xi=\sum_{i} \frac{x_{\imath}^{2}+y_{i}^{2}}{\eta-c_{i}}
$$


Now let

$$
H(x, y)=\frac{1}{2 \pi i} \int_{|\eta|=R} f(\eta) \phi_{n}(x, y) d \eta
$$

for suitable $f$, where $R$ contains the spectrum of $C$. Putting

$$
\phi_{n}(x, y)=\sum_{j=1}^{n} \frac{G_{j}(x, y)}{z-C_{j}}, \quad G_{j}(x, y)=\left|z_{j}\right|^{2}
$$

we get

$$
H(x, y)=\frac{1}{2} \sum_{j=1}^{n} f\left(c_{\jmath}\right) G_{j}(x, y) .
$$

Choosing $f$ so $f\left(c_{j}\right)=c_{\jmath}$, we get

$$
H=\frac{1}{2} \sum_{j=1}^{n} c_{\jmath}\left(x_{k}^{2}+y_{k}^{2}\right) .
$$

Then the equations of motion are simply

$$
\dot{x}=\frac{\partial H}{\partial y}, \quad \dot{y}=-\frac{\partial H}{\partial x},
$$

which give the required result.

Note also that, via Moser, we can get an independent derivation of the Lax pair (see [11]).

5. The flow has the following statistical interpretation: It reflects the asymmetries in the distribution of data points or the relative importance of the principal components in a principal component analysis; it is a measure of sphericity (see $[4,12]$ ).

Finally, we note that we have added a system to the list of completely integrable "spinning top and ellipsoid" type systems (see [1] ), where the matrix $L(x, y)$ takes the form

(a) $\alpha \xi+\Gamma_{x y}(N$-dimensional free rigid body),

(b) $\alpha \xi^{2}+\xi \Gamma_{x y}-\Gamma_{x x}$ (geodesic flow on ellipsoid, Neumann's Problem),

(c) $\alpha \xi^{2}+\xi \Gamma_{x y}+(x \otimes y+y \otimes x-\alpha)$ (central force problem on ellipsoid). To this list we have added

(d) $\alpha \xi+\Gamma_{x x}+\Gamma_{y y}+i \Gamma_{x y}$ (line fitting problem).

I would like to thank Professor C. I. Byrnes for his valuable advice and comments.

Acknowledgement. I would like to thank Professor C. I. Byrnes for his valuable advice and comments.

\section{REFERENCES}

1. M. Adler and P. Van Moerbeke, Completely integrable systems, Euclidean Lie algebras and curves, Adv. in Math. 38 (1980), 267-317.

2. M. F. Atiyah, Convexity and commuting Hamiltonians, Bull. London Math. Soc. 14 (1982), 1-15.

3. , Angular momentum, convex polyhedra and algebraic geometry, Proc. Edinburgh Math. Soc. 26 (1983), 121-138. 
4. A. M. Bloch, Ph.D. Thesis, Harvard Univ. (in preparation).

5. C. I. Byrnes and J. C. Willems, Least squares estimation, linear programming and momentum (to appear).

6. G. H. Golub and C. F. Van Loan, An analysis of the total least squares problem, SIAM J. Numer. Anal. 17 (1980), 883-893.

7. P. A. Griffiths, Linearizing flows and a cohomological interpretation of the Lax equation (preprint).

8. D. Kendall, Multivariate analysis, Macmillan, 1975.

9. H. P. McKean, Integrable systems and algebraic curves, Lecture Notes in Math., vol. 755, Springer-Verlag, 1978, pp. 83-200.

10. A. S. Mischenko and A. T. Fomenko, Integrability of Euler equations on semisimple Lie algebras, Selecta Math. Soviet. 2 (1982), 207-292.

11. J. Moser, The geometry of quadrics and spectral theory, Proc. Chern Sympos. (1979), Springer-Verlag, 1980.

12. R. S. Muirbead, Aspects of multivariate statistical theory, Wiley, 1982.

13. D. Mumford, Tata lectures on Theta II, Birkhauser, 1984.

14. T. Raitu, Involution theorems, Lecture Notes in Math., vol. 775, Springer-Verlag, 1980, pp. 221-257.

15. , The motion of the free n-dimensional rigid body, Indiana Univ. Math. J. 29 (1980), 609-629.

Division OF APPlied SCIENCES, HARVARD UNIVERSITY, CAMBRIDGe, MASSACHUSETTS 02138 